\title{
Constructing Multiple Light Multicast Trees in WDM Optical Networks
}

\author{
Weifa Liang \\ Department of Computer Science \\ Australian National University \\ Canberra, ACT 0200, Australia \\ wliang@Cs.anu.edu.au
}

\begin{abstract}
Multicast routing is a fundamental problem in any telecommunication network. We address the multicast issue in a WDM optical network without wavelength conversion capability. To realize a multicast request in such network, it is required to establish multiple light multicast trees (MLMT) sometimes due to the fact that the requested network resources are being occupied by other routing traffic and therefore it is impossible to establish a single light multicast tree for the request. In this paper we first propose a multiple light multicast tree model. We then show the MLMT is NP-hard, and devise an approximation algorithm for it which takes $O\left((k n)^{1 / \epsilon}|D|^{2 / \epsilon}+k n+k m\right)$ time and delivers an approximation solution within $O\left(|D|^{\epsilon}\right)$ times of the optimal, where $n, m$, and $k$ are the numbers of nodes, links, and wavelengths in the network, $D$ is the set of destination nodes and $\epsilon$ is constant, $0<\epsilon \leq 1$. We finally extend the problem further with the end-to-end path delay is bounded by an integer $\Delta$, and we call this latter problem as the multiple delay-constrained light multicast tree problem (MDCLMT), for which we propose two approximation algorithms with the performance ratios of $|D|$. One of the proposed algorithms takes $O\left(k m|D| \Delta+|D|^{2} m \Delta\right)$ time and the path delay is strictly met; and another takes $O\left(\mathrm{kmn} / \epsilon+|D| n^{2}\right)$ time and the path delay is no more than $(1+\epsilon) \Delta$, where $\epsilon$ is constant with $0<\epsilon \leq 1$.
\end{abstract}

\section{Introduction}

Wavelength-division-multiplexing (WDM) is emerging as a key technology for next-generation networks by providing unprecedented bandwidth in a medium that is free from inductive and capacitive loadings, thus, relaxing the limitations imposed on the bandwidth-distance product. In WDM optical networks the fibre bandwidth is partitioned into multiple data channels which may be transmitted simultaneously on different wavelengths. In this paper we consider circuit-switched WDM networks, which can be further classified as either single-hop or multi-hop networks [10,11]. In single-hop networks each message is transmitted from the source to the destination without any optical-to-electronic conversion within the network. Therefore, single-hop communication can be realized by using a single wavelength to establish a connection, but such connections may in general be difficult or impossible to find in the presence of other network traffic. Alternatively, all-optical wavelength converters may be used to convert from one wavelength to another wavelength within the network. However, such converters are likely to prohibitively expensive for most applications in the foreseeable future [15]. In this paper we therefore only consider the single-hop WDM optical network.

Multicast is a fundamental problem in telecommunication networks, which arises in a wide variety of applications such as video conferencing, entertainment distribution, teleclassrooms, distributed data processing, etc [12]. In WDM networks the multicast problem is also referred to one-tomany routing and wavelength assignment problem (RWA), which aims at finding a set of links and wavelengths on these links to establish the connection from the source to the destination nodes.

Lots of effort for multicast in multi-hop networks has been taken in past years. The cost of using the network resources in such networks is measured as follows. A wavelength traversing a link incurs a cost and the wavelength conversion at a node also incurs a cost. Thus, the multicast problem is to find a multicast tree rooted at the source and spanning the destination nodes in $D$ such that the tree cost is minimized [6], where $D$ is the set of destination nodes. When the size $|D|$ of the set of destination nodes is 1 , the multicast problem becomes the optimal semilightpath problem [2], for which Chlamtac et al [2] presented an $O\left(k^{2} n+k n^{2}\right)$ time algorithm for it, where $k$ is the number of wavelengths and $n$ is the number of nodes in the network. Liang and Shen [7] later provided an improved algorithm, which takes $O\left(k^{2} n+k m+k n \log (k n)\right)$ time and can be implemented in the distributed environment effi- 
ciently, where $m$ is the number of links in the network. For $D \subset V$, Liang and Shen [6] proposed an approximation algorithm for finding a multicast tree with minimizing the tree cost. Sahin and Azizolgu [14] considered the multicast problem under various fanout polices and Malli et al [9] dealt with this problem under a sparse splitting model. Sahasrabuddhe and Mukherjee [13] formulated the problem as a mixed-integer linear programming problem. Znati et al [17] dealt with the problem by decoupling the delay from the cost model, and presented several heuristic algorithms for finding a multicast tree under the constraint of end-toend delay from the source to the destination nodes. In addition, there have been several other studies for constructing constrained multicast trees in the WDM optical networks. For example, Libeskind-Hadas [8] proposed a multi-path routing model, in which the multicast problem is to find a set of paths from the source to the destination nodes such that each path contains a subset of destination nodes, the nodes in the set of destination nodes are included by these paths, and the cost sum of these paths is minimized. Zhang et al [16] considered the multicast problem by focusing on the limited splitting power of optical switches and provided four heuristic algorithms for the problem.

Due to currently it is prohibitively expensive to employ the wavelength conversion switches in optical networks, in this paper we consider a circuit-switched single-hop WDM optical network. We extend the multi-path routing concept [8] further by proposing a multiple light multicast tree model, on which we deal with the multicast problem. Specifically, given a source $s$ and a set of destination nodes $D$, if the wavelength conversion at nodes is not allowed, then it is impossible to realize a multicast request through the construction of a single light multicast tree rooted at the source including all the nodes in $D$ due to lack of the available resources that are being used by other routing traffics. Instead, to realize a multicast request, it necessitates to construct several light multicast trees that cover the nodes in $D$, here a light multicast tree is referred to a tree in which every link has the same wavelength. To minimize the network resource consumption for a multicast session, the objective is to minimize the cost summation of all the light multicast trees. We thus refer to the multicast problem on this cost model as the multiple light multicast tree problem (MLMT for short). When the set of destination nodes includes all other nodes except the source $s$, the problem becomes a broadcast problem which is called the light broadcast tree problem. If the end-to-end delay of a routing path in each of the trees from the root (source) to a destination node is bounded by an integer $\Delta$, then the problem is referred to the multiple delay-constrained light multicast tree problem (MDCLMT for short). Under our model we first show the mulicast problem (MLMT) is NP-Complete even when the set $D$ of destination nodes is $D=V-\{s\}$. We then provide an approximation algorithm for it which takes $O\left((k n)^{1 / \epsilon}|D|^{2 / \epsilon}+k n+k m\right)$ time and delivers a solution within $O\left(|D|^{\epsilon}\right)$ times of the optimal, where $\epsilon$ is constant, $0<\epsilon \leq 1$. We finally deal with the MDCLMT, for which two approximation algorithms with performance ratios of $|D|$ are proposed, which trade-offs between the running time and the accuracy of the path delay. One of the proposed algorithms takes $O\left(k m|D| \Delta+|D|^{2} m \Delta\right)$ time and the path delay is strictly met; and another takes $O\left(k m n / \epsilon+|D| n^{2}\right)$ time which is independent of $\Delta$, and the path delay is no more than $(1+\epsilon) \Delta$, where $\epsilon$ is constant with $0<\epsilon \leq 1$.

The rest of the paper is organized as follows. In Section 2 notations are introduced and the problems are defined. In Section 3 the MLMT is shown to be NP-Complete even when the destination set is $D=V-\{s\}$, an approximation algorithm for it is proposed, and the running time as well the performance ratio of the algorithm is analyzed. In Section 4 the MDCLMT is considered, and two approximation algorithms for it with performance ratios of $|D|$ are presented.

\section{Preliminaries}

The network model: The optical network is modeled by a directed graph $G=(V, E, \Lambda)$, where $V$ is a set of nodes (vertices) representing switches, $E$ is a set of directed links (edges) representing the optical fibers, and $\Lambda$ is a set of wavelengths in $G, n=|V|, m=|E|$, and $|\Lambda|=k$. Let $\Lambda=\left\{\lambda_{1}, \lambda_{2}, \ldots, \lambda_{k}\right\}$. Associated with each link $e \in E$, a wavelength set $\Lambda(e)(\subseteq \Lambda)$ is given, and for each $\lambda \in \Lambda(e)$, a non-negative weight $w(e, \lambda)$ is assigned which is the cost of traversing $e$ using wavelength $\lambda$. This cost reflects the communication bandwidth consumption on $e$. Sometimes, the routing congestion factor on the link is also incorporated in the cost by assigning different weights to different wavelengths. In some cases it is also assumed that an integral delay $\delta(e)$ is associated with each link $e \in E$.

Problem formulation: A multicast request is an ordered pair $(s ; D)$ where $s \in V$ is the source of the multicast session and $D(\subseteq V-\{s\})$ is the set of destination nodes. Assume that multicast requests are made and released dynamically. To realize a multicast communication request, the ideal case is to establish a single light multicast tree. However, due to the fact that some specific network resource, e.g., a specific wavelength, is occupying by other communication traffic at this moment, and it is impossible to establish such a tree for the current multicast request. Instead, it is required to establish a collection of light multicast trees to realize the request. Specifically, we deal with the following two multicast problems.

The Multiple Light Multicast Tree Problem (MLMT) is to construct a collection of multicast trees $T_{1}, T_{2}, \ldots T_{k^{\prime}}$ such that (i) tree $T_{j}$ is a light multicast tree rooted at $s$ 
including a subset of nodes in $D$, and wavelength $\lambda_{i_{j}}$ is on each link of $T_{j}, 1 \leq i_{j} \leq k, 1 \leq j \leq k^{\prime}$; (ii) each node in $D$ as a leaf node is included in one of the $k^{\prime}$ trees at most once but it may serve as a relay node in other trees. The union of the nodes in the $k^{\prime}$ trees satisfies that $D \subseteq \cup_{i=1}^{k^{\prime}} V\left(T_{i}\right)$; (iii) $\sum_{j=1}^{k^{\prime}} C\left(T_{j}\right)$ is minimized, where $C\left(T_{j}\right)=\sum_{e \in E\left(T_{j}\right)} w\left(e, \lambda_{i_{j}}\right)$. Clearly $k^{\prime} \leq|\Lambda|=k$.

In some real-time QoS applications, not only the cost of multicast trees is the main optimization objective, but also the response time (the path delay) from the source to each destination node in a multicast session is paramount. For this latter case, another extra metric - an integral delay $\delta(e)$ is assigned, in addition to the cost metric for each link $e \in$ $E$. Thus, given a source $s$, an integral delay $\Delta$, and a set of destination nodes $D$, the Multiple Delay-Constrained Light Multicast Tree Problem (MDCLMT) is to find a collection of light multicast trees $T_{1}, T_{2}, \ldots, T_{k^{\prime}}$ such that (i) tree $T_{j}$ is a light multicast tree rooted at $s$ covering a subset of $D$, and the wavelength $\lambda_{i_{j}}$ is on each link of $T_{j}, 1 \leq i_{j} \leq k$, $1 \leq j \leq k^{\prime}$; (ii) each node $v \in D$ as a leaf node is included in one of the $k^{\prime}$ trees at most once but it may appear as a relay node in other trees, and the path delay in the tree from $s$ to $v$ is bounded by $\Delta$; (iii) $\sum_{j=1}^{k^{\prime}} C\left(T_{j}\right)$ is minimized, where $C\left(T_{j}\right)=\sum_{e \in E\left(T_{j}\right)} w\left(e, \lambda_{i_{j}}\right)$.

\section{Approximation Algorithm for the MLMT}

\subsection{The Light Broadcast Tree Problem is NP- Hard}

When the destination set $D=V-\{s\}$, the MLMT becomes the light broadcast tree problem. In contrast to that the broadcast tree problem (the minimum spanning tree) in conventional networks is polynomially solvable, the light broadcast tree problem in WDM optical networks on the multiple tree model is NP-Complete. We state it in the following theorem without proof due to the space limit. The major technique is to reduce the 3-CNF SAT problem to it.

Theorem 1 The light broadcast tree problem in WDM networks on the multiple light multicast tree model is NPComplete.

\subsection{Approximation Algorithm}

We have shown that the light broadcast tree problem is NP hard, so is the MLMT. We thus focus on devising an approximation algorithm for it. The basic idea is to transform the problem to a directed Steiner tree problem in an auxiliary directed graph $G C$. As a result, a feasible solution for this latter problem will give a feasible solution for the concerned problem.

For each distinct wavelength $\lambda_{i} \in \Lambda$, a subgraph $G_{\lambda_{i}}$ of $G$ containing $s$ is induced by the links that the wave- length $\lambda_{i}$ is on them and every other node in the subgraph is reachable from $s$. Let $G_{\lambda_{i}}\left(V_{i}, E_{i}\right)$ be the subgraph and let node $v$ be included in $G_{\lambda_{i}}$, then $v$ in $G_{\lambda_{i}}$ is relabeled as $\left(v, \lambda_{i}\right)$. The weight of a corresponding link $e^{\prime}$ in $G_{\lambda_{i}}$ is $w\left(e, \lambda_{i}\right)$ if $e=\langle u, v\rangle \in E$ and $u$ is reachable from $s$ and $\lambda_{i} \in \Lambda(e)$, then $e^{\prime}=\left\langle\left(u, \lambda_{i}\right),\left(v, \lambda_{i}\right)\right\rangle \in E_{i}, 1 \leq i \leq k$. Then, the auxiliary graph $G C(V C, E C)$ is constructed as follows. $\quad V C=\bigcup_{i=1}^{k} V_{i} \bigcup\left\{s^{\prime}\right\} \bigcup\left\{v^{\prime} \mid v \in D\right\}$ and $E C=\bigcup_{i=1}^{k} E_{i} \bigcup_{i=1}^{k}\left\{\left\langle s^{\prime},\left(s, \lambda_{i}\right)\right\rangle\right\} \bigcup\left\{\left\langle\left(v, \lambda_{i}\right), v^{\prime}\right\rangle \mid v \in\right.$ $D\}$, where $s^{\prime}$ is a virtual node representing the source $s, v^{\prime}$ represents the node $v \in D$, the set of wavelengths on link $\left\langle s^{\prime},\left(s, \lambda_{i}\right)\right\rangle$ in $G C$ is $\left\{\lambda_{i}\right\}$, and the set of wavelengths on link $\left\langle\left(v, \lambda_{i}\right), v^{\prime}\right\rangle$ in $G C$ is $\left\{\lambda_{i}\right\}$. The weight assignment of links in $G C$ is defined as follows. For each link $e \in E_{i}$, its weight in $G C$ is $w\left(e, \lambda_{i}\right)$, for each link $\left\langle s^{\prime},\left(s, \lambda_{i}\right)\right\rangle$, its weight is $w\left(\left\langle s^{\prime},\left(s, \lambda_{i}\right)\right\rangle, \lambda_{i}\right)=0$, and for each $v \in D$, the weight of link $\left\langle\left(v, \lambda_{i}\right), v^{\prime}\right\rangle$ is $w\left(\left\langle\left(v, \lambda_{i}\right), v^{\prime}\right\rangle, \lambda_{i}\right)=0$. The auxiliary graph $G C$ has the following properties. (i) For each node $v \in D$, there is at least a directed path in $G C$ from $s^{\prime}$ to $v^{\prime}$, using the links in a specific $G_{\lambda_{i}}$, links $\left\langle s^{\prime},\left(s, \lambda_{i}\right)\right\rangle$ and $\left\langle\left(v, \lambda_{i}\right), v^{\prime}\right\rangle$ in $G C$. (ii) Each $G_{\lambda_{i}}$ is a subgraph of $G C$. The nodes in $G C$ between $G_{\lambda_{i}}$ and $G_{\lambda_{j}}$ are not reachable from each other, if $i \neq j$. We then have the following theorem.

Theorem 2 The multicast tree in GC rooted at $s^{\prime}$ including the nodes in $\left\{v^{\prime} \mid v \in D\right\}$ corresponds to an optimal solution for the MLMT in G.

Proof Let $F_{o p t}$ be an optimal solution for the MLMT in $G$ consisting of the collection of light multicast trees and $C\left(F_{\text {opt }}\right)$ be the cost sum of the light multicast trees in $F_{\text {opt }}$. Following the construction of $G C$, it is easy to show that there is a corresponding multicast tree $T C$ in $G C$ in which each tree $T_{j}$ in $F_{\text {opt }}$ corresponds to a subtree rooted at $\left(s, \lambda_{i_{j}}\right)$ of $T C$, and $T C$ is a multicast tree rooted at $s^{\prime}$ including the nodes in $\left\{v^{\prime} \mid v \in D\right\}$. Let $w(T C)$ be the weighted sum of the links in $T C$. Then, $w(T C)=C\left(F_{\text {opt }}\right)$.

Assume that $T_{\text {opt }}^{G C}$ is a directed Steiner tree in $G C$ rooted at $s^{\prime}$ including the nodes in $\left\{v^{\prime} \mid v \in D\right\}$. Following the definition of directed Steiner trees, the weighted sum $w\left(T_{o p t}^{G C}\right)$ of the links in $T_{o p t}^{G C}$ is $w\left(T_{o p t}^{G C}\right) \leq w(T C)=C\left(F_{\text {opt }}\right)$. The theorem follows. $\square$

It is well known that the directed Steiner tree problem is NP hard [3]. In the following we aim to find an approximate, directed Steiner tree in $G C$ rooted at $s^{\prime}$ including the nodes in $\left\{v^{\prime} \mid v \in D\right\}$, which in turn will give an approximation solution for the MLMT. The detailed algorithm (Fig 1) is described as follows.

Theorem 3 Given a directed network $G=(V, E, \Lambda)$ with a given source $s$ and a node set $D$, there is an approximation algorithm for the MLMT with a performance ratio of 
Algorithm App_Multicast_Trees

1. Construct an auxiliary graph $G C(V C, E C)$;

2. Find an approximate directed Steiner tree $T C_{a p p}^{G C}$ in $G C$ rooted at $s^{\prime}$ including the nodes in $\left\{v^{\prime} \mid v \in D\right\}$;

3. Tree $T_{i}$ corresponds to a light multicast tree in $G$ with $\lambda_{i}$, and a node $v \in D$ in included in the multicast tree if node $\left(v^{\prime}, \lambda_{i}\right)$ is included in $T_{i}, 1 \leq i \leq k$.

\section{Figure 1. Algorithm for the MLMT}

$O\left(|D|^{\epsilon}\right)$. The time complexity of the proposed algorithm is $O\left((k n)^{1 / \epsilon}|D|^{2 / \epsilon}+k n+k m\right)$, where $\epsilon$ is constant with $0<\epsilon \leq 1$.

Proof Following the construction of $G C$, it contains at most $k n+|D|+1$ nodes and $k m+k|D|+k$ links. Thus, Step 1 takes $O(k m+k n)$ time obviously. Step 2 is to find an approximate, directed Steiner tree $T_{a p p}^{G C}$ in $G C$ rooted at $s^{\prime}$ including the nodes in $\left\{v^{\prime} \mid v \in D\right\}$, which takes $O\left((k n)^{1 / \epsilon}|D|^{2 / \epsilon}\right)$ time, using the approximation algorithm in [1], where $\epsilon$ is a constant with $0<\epsilon \leq 1$. Step 3 takes $O(k n)$ time because the number of links in $T_{a p p}^{G C}$ is bounded by $O(k n+|D|)$. The cost of $T_{a p p}^{G C}$ therefore is $w\left(T_{a p p}^{G C}\right) \leq$ $c|D|{ }^{\epsilon} w\left(T C_{o p t}^{G C}\right)=c|D|^{\epsilon} w(T C)=c|D|{ }^{\epsilon} C\left(F_{\text {opt }}\right)$, by Theorem 2, where $c$ and $\epsilon$ are constants and $0<\epsilon \leq 1$.

\section{Approximation Algorithm for the MD- CLMT}

In this section we start with a routine for finding a delayconstrained shortest path in a graph bounded by an end-toend integral delay $\Delta$, which will be used in the proposed algorithms. We then provide approximation algorithms for the MDCLMT.

\subsection{The delay-constrained shortest path problem}

The delay-constrained shortest path problem is to find a shortest path in $G$ from $s$ to $t$ under the constraint that the end-to-end delay of the path is bounded by $\Delta$. If the path delay $\Delta$ is not an integer, the problem is NP-hard [3]; otherwise, it is polynomial solvable, and one such a dynamic programming algorithm due to Kompella et al [5] is described as follows.

Let $C_{d}(u, v)$ be the cost of a shortest path from $u$ to $v$ with the delay exactly $d$ and $C(u, v)$ be the cost of the shortest path in $G$ with a bounded delay $\Delta$. If there are multiple shortest paths with the same cost, then the one with the least delay is chosen. Thus, $C_{d}(u, v)$ and $C(u, v)$ are formulated as follows. $C_{d}(u, v)=\min _{x \in V}\left\{C_{d-\delta(x, v)}(u, x)+\right.$ $w(x, v)\}$ and $C(u, v)=\min _{d \leq \Delta}\left\{C_{d}(u, v)\right\}$. Then, the solution for the problem is to find the value of $C(s, t)$. Use Dijkstra's algorithm, it takes $O(\Delta m)$ time to find a solution for this dynamic programming problem.

Let $P(s, t, \lambda)$ be a delay-constrained shortest path in a subgraph $G_{\lambda}$ of $G$ from $s$ to $t$ with the end-toend delay $\Delta$, the cost of $P(s, t, \lambda)$ is $C(P(s, t, \lambda))=$ $\sum_{e \in P(s, t, \lambda)} w(e, \lambda)$, where the subgraph $G_{\lambda}$ is induced by the links in $G$ containing wavelength $\lambda$ and $e$ is a link in $P(s, t, \lambda)$. It is easy to see that the dynamic programming algorithm for the delay-constrained shortest path problem is a pseudo-polynomial algorithm, because its running time depends on the delay $\Delta$. However, if the end-to-end delay is not met strictly, there is a strongly polynomial approximation algorithm for this problem, which is stated as follows.

Lemma 1 Given a WDM optical network $G(V, E, \Lambda)$ with a pair of nodes $s$ and $t$, and an integral delay $\Delta$, assume that each link $e$ has been assigned a set $\Lambda(e)(\subseteq \Lambda)$ of wavelengths, each wavelength $\lambda \in \Lambda(e)$ traversing e incurs a cost $w(e, \lambda)$, and the delay $\delta(e)$ for traversing on $e$ is an integer and $\delta(e) \leq \Delta$. There is an $O(m n / \epsilon)$ approximation algorithm for finding a delay-constrained shortest path from $s$ to $t$ with the relaxation of the bounded delay, which finds a shortest path with the bounded delay $(1+\epsilon) \Delta$, where $\epsilon$ is constant, $0<\epsilon \leq 1$.

Proof The approximation algorithm [4] for the delayconstrained shortest path problem uses a scaling technique, which delivers an optimal solution in terms of the path cost but the path delay is bounded by $(1+\epsilon) \Delta$, where $\epsilon$ is constant, $0<\epsilon \leq 1$.

\subsection{Approximation algorithm with strictly delay}

We here propose an approximation algorithm for the MDCLMT such that the end-to-end delay of the routing paths must be met strictly. The idea behind the proposed algorithm is as follows. Let the approximation solution consist of $k^{\prime}$ light multicast trees $T_{1}, T_{2}, \ldots, T_{k^{\prime}}$, where wavelength $\lambda_{i_{j}}$ is on each link of $T_{j}, 1 \leq j \leq k^{\prime}$, $1 \leq i_{j} \leq k$. For each node $v \in D, v$ is included in $T_{j}$ if $C\left(P\left(s, v, \lambda_{i_{j}}\right)\right) \leq C\left(P\left(s, v, \lambda_{i_{j^{\prime}}}\right)\right)$ for all $j^{\prime} \neq j$. Let $D_{i_{j}}$ be the subset of $D$ whose nodes are included in $T_{i_{j}}$, then $\cup_{j=1}^{k^{\prime}} D_{i_{j}}=D, 1 \leq j \leq k^{\prime} \leq k$ and $1 \leq i_{j} \leq k$. Thus, we have the following important lemma.

Lemma 2 Let $C^{*}$ be the cost of an optimal solution for the MDCLMT. Then, $C\left(P\left(s, v, \lambda_{i_{j}}\right)\right) \leq C^{*}$.

Proof Assume that the optimal solution for the MDCLMT consists of $k^{*}$ light multicast trees $T_{1}^{*}, T_{2}^{*}, \ldots, T_{k^{*}}^{*}$, where wavelength $\lambda_{l_{j}}$ is on every link of $T_{j}^{*}, 1 \leq l_{j} \leq k$ and $1 \leq j \leq k^{*} \leq k$. Then, $\sum_{j=1}^{k^{*}} C\left(T_{j}^{*}\right)=C^{*}$. If 
$v \in D$ is included in $T_{x}^{*}$ in the optimal solution, then, $C\left(P\left(s, v, \lambda_{l_{x}}\right)\right) \leq C\left(T_{x}^{*}\right)$. While $v$ is included in $G_{\lambda_{i_{j}}}$, we thus have $C\left(P\left(s, v, \lambda_{i_{j}}\right)\right) \leq C\left(P\left(s, v, \lambda_{l_{x}}\right)\right) \leq C\left(T_{x}^{*}\right) \leq$ $C^{*}$.

We thus have an approximation algorithm (See Fig. 2) for the problem.

Algorithm App_Constrained_Multicast_Trees

1. for $i \leftarrow 1$ to $k$ do

2. for each node $v \in D$ do

3. find a delay-constrained shortest path $P\left(s, v, \lambda_{i}\right)$ endfor;

in $G_{\lambda_{i}}$ from $s$ to $v$;

endfor;

4. partition the nodes in $D$ into $k^{\prime}$ disjoint subsets such that a node $v \in D$ is also in $D_{i_{j}}$

if $C\left(P\left(s, v, \lambda_{i_{j}}\right)\right)=\min _{1 \leq i \leq k}\left\{C\left(P\left(s, v, \lambda_{i}\right)\right)\right\}$

5. for $\leftarrow \leftarrow 1$ to $k^{\prime}$ do

6. find a delay-constrained multicast tree $T_{j}$ in $G_{\lambda_{i_{j}}}$ rooted at $s$ including nodes in $D_{i_{j}}$.

endfor;

7. A feasible solution for the problem is $\left\{T_{1}, T_{2}, \ldots, T_{k^{\prime}}\right\}$ since $D=\cup_{j=1}^{k^{\prime}} D_{i_{j}} \subset \cup_{j=1}^{k^{\prime}} V\left(T_{j}\right)$.

\section{Figure 2. Algorithm for the MDCLMT}

Theorem 4 Given a directed network $G=(V, E, \Lambda)$ with a given source $s$ and $a$ node set $D$ and an integral delay $\Delta$, assume that each link $e \in E$ has been assigned a set $\Lambda(e)(\subseteq \Lambda)$ of wavelengths, each wavelength $\lambda \in \Lambda(e)$ traversing $e$ incurs a cost $w(e, \lambda)$, and the delay $\delta(e)(\leq \Delta)$ for traversing $e$ is an integer. There is an approximation algorithm for the MDCLMT with a performance ratio of $|D|$. The time complexity of the proposed algorithm is $O\left(\left(k m|D| \Delta+|D|^{2} m \Delta\right)\right.$.

Proof Let $C^{*}$ be the cost of the optimal solution for the MDCLMT. Then, following Lemma 2, $C\left(P\left(s, v, \lambda_{i_{j}}\right)\right) \leq$ $C^{*}$ for each $v \in D_{i_{j}}$. As a result, the cost $C\left(T_{j}\right)$ of $T_{j}$ is $C\left(T_{j}\right) \leq\left|D_{i_{j}}\right| C^{*}, 1 \leq j \leq k^{\prime}$. Thus, the total cost of the $k^{\prime}$ light multicast trees is $\sum_{1 \leq j \leq k^{\prime}} C\left(T_{j}\right) \leq$ $\sum_{1<j \leq k^{\prime}}\left|D_{i_{j}}\right| C^{*}=|D| C^{*}$.

The running time from Step 1 to Step 4 is $O\left(k|D| T_{s s p}(\Delta)\right)=O(k m|D| \Delta)$, where $T_{s s p}(\Delta)$ is the time for finding a delay-constrained shortest path from $s$ to $v$ in a graph with $n$ nodes and $m$ links with the end-to-end delay $\Delta$. Step 6 takes $O\left(\left|D_{i_{j}}\right|^{2} T_{s s p}(\Delta)\right)$ time [5], and the solution delivered is $\left|D_{i_{j}}\right|$ times of the optimal. So, the running time for Steps 5 and 6 is $\sum_{1 \leq j \leq k^{\prime}} O\left(\left|D_{i_{j}}\right|^{2} T_{s s p}(\Delta)\right)=O\left(|D|^{2} m \Delta\right)$. The algorithm therefore takes $O\left(k m|D| \Delta+|D|^{2} m \Delta\right)$ time.

\subsection{Approximation algorithm without strictly de- lay}

We now provide a truly polynomial approximation algorithm for the MDCLMT if the end-to-end delay of a path is not met strictly. The basic idea is similar to the one in the preceding section except the following modifications. At Step 2 an approximation algorithm instead of an exact algorithm for finding a delay-constrained shortest path will be used. The set $D$ of destination nodes is then partitioned into $k^{\prime}$ disjoint subsets $D_{i_{j}}, 1 \leq j \leq k^{\prime}$ and $1 \leq i_{j} \leq k$. For each subset $D_{i_{j}}$, at Step 6 a new approach called sticking approach instead of the algorithm due to Kompella et al [5] will be employed to find an approximate, light multicast tree in $G_{\lambda_{i_{j}}}$ rooted at $s$ including all nodes in $D_{i_{j}}$. In the following we present the sticking approach.

Let $P_{a p p}(s, v, \lambda)$ be a shortest path in $G_{\lambda}$ from $s$ to $t$ with a bounded delay no more than $(1+\epsilon) \Delta$, founded by applying the Goel et al [4] algorithm on $G_{\lambda}$. A node $v \in D$ is included in set $D_{i_{j}}$ if and only if $C\left(P_{a p p}\left(s, v, \lambda_{i_{j}}\right)\right) \leq C\left(P_{a p p}(s, v, \lambda)\right)$ for any other $\lambda \in \Lambda$. Let $l=\left|D_{i_{j}}\right|$ and $v_{1}, v_{2}, \ldots, v_{l}$ be the sequence of nodes in $D_{i_{j}}$ sorted in increasing order, i.e, $C\left(P_{a p p}\left(s, v_{1}, \lambda_{i_{j}}\right)\right) \leq$ $C\left(P_{a p p}\left(s, v_{2}, \lambda_{i_{j}}\right)\right) \leq \ldots \leq C\left(P_{a p p}\left(s, v_{l}, \lambda_{i_{j}}\right)\right)$. The sticking approach is to construct a light multicast tree including the nodes in $D_{i_{j}}$ by adding these $l$ paths one by one until all nodes in $D_{i_{j}}$ are included.

Assume that a light multicast tree $T P_{i-1}$ rooted at $s$ including nodes in $\left\{v_{1}, v_{2}, \ldots, v_{i-1}\right\}, i<l$ has already been constructed. Now consider adding the path $\left.P_{a p p}\left(s, v_{i}, \lambda_{i_{j}}\right)\right)$ to $T P_{i-1}$ to form a new light multicast tree $T P_{i}$ including the nodes in $\left\{v_{1}, v_{2}, \ldots, v_{i}\right\}$ such that the path delay in $T P_{i}$ from $s$ to each node $v_{j}$ is within $(1+\epsilon) \Delta, 1 \leq j \leq i$.

Initially $T P_{1}=P_{a p p}\left(s, v_{1}, \lambda_{i_{j}}\right)$ and $i=1$. Consider adding a path $P_{i}=P_{a p p}\left(s, v_{i}, \lambda_{i_{j}}\right)$ to $T P_{i-1}$ to construct $T P_{i}$ for $i>1$. Let $P_{i}(x, y)$ be a segment of path $P_{i}$ starting at node $x$ and finishing at node $y$. We traverse $P_{i}$ from the source $s$. Let $u_{r}$ be the $r$ th node in $P_{i}$ appeared in both $T P_{i-1}$ and $P_{i}$ except the source $s$. Thus, path $P_{i}$ can be partitioned into $r$ segments if $r$ nodes appear in both $T P_{i-1}$ and $P_{i}$. Clearly, $r \geq 0$. Let $T P_{i}^{r}$ be the tree after having considered the $r$ th segment $P_{i}\left(u_{r-1}, u_{r}\right)$ of $P_{i}$ to $T P_{i-1}^{r-1}$, where $T P_{i-1}^{r-1}$ is the resulting tree after having considered the previous $(r-1)$ segments of $P_{i}$. Denote by $T P_{i-1}^{r-1}(x, y)$ the unique path in $T P_{i-1}^{r-1}$ from $x$ to $y$. If $r=0$, stick the entire path $P_{i}$ to $T P_{i-1}$ to form a new tree $T P_{i}$. Otherwise, $T P_{i}$ is constructed by taking into account the segments of $P_{i}$ one by one, which is described as follows. Let $u_{1}$ be the first node in $P_{i}$ which is also in $T P_{i-1}$. Following the definition, we have $T P_{i-1}^{0}=T P_{i-1}$. Then, there are two node-disjoint paths in $T P_{i-1}^{0} \cup\left\{P_{i}\left(s, u_{1}\right)\right\}$ from $s$ to $u_{1}$ when the segment $P_{i}\left(s, u_{1}\right)$ is added to $T P_{i-1}^{0}$. Among the two paths, one is $T P_{i-1}^{0}\left(s, u_{1}\right)$ consisting of tree edges 
only; and another is $P_{i}\left(s, u_{1}\right)$ of $P_{i}$. Let $D(P)$ be the delay of a path $P$. If $D\left(T P_{i-1}^{0}\left(s, u_{1}\right)\right) \leq D\left(P_{i}\left(s, u_{1}\right)\right)$, then we just ignore $P_{i}\left(s, u_{1}\right)$ of $P_{i}$ for any further consideration, and the path delay in $T P_{i-1}^{0}$ from $s$ to $v_{i}$ is no greater than $(1+\epsilon) \Delta$ because $D\left(T P_{i-1}^{0}\left(s, u_{1}\right)\right)+D\left(P_{i}\left(u_{1}, v_{i}\right)\right) \leq$ $D\left(P_{i}\left(s, u_{1}\right)\right)+D\left(P_{i}\left(u_{1}, v_{i}\right)\right) \leq(1+\epsilon) \Delta$. Otherwise, we delete the tree edge $\left(u_{1}\right.$, parent $\left.\left(u_{1}\right)\right)$ from $T P_{i-1}^{0}$, where parent $(v)$ is the parent of $v$ in $T P_{i-1}^{0}$. Then, the path delay in $T P_{i-1}^{1}$ from $s$ to each leaf node $v_{j}$ which is included in the subtree rooted at $u_{1}$ is no more than $(1+\epsilon) \Delta$, because $D\left(P_{i}\left(s, u_{1}\right)\right)+D\left(T P_{i-1}^{0}\left(u_{1}, v_{j}\right)\right) \leq D\left(T P_{i-1}^{0}\left(s, u_{1}\right)\right)+$ $D\left(T P_{i-1}^{0}\left(u_{1}, v_{i}\right)\right) \leq(1+\epsilon) \Delta, 1 \leq j \leq(i-1)$.

Assume that the first $(r-1)$ segments of $P_{i}$ have been considered and the resulting tree is $T P_{i-1}^{r-1}$. Now we consider adding the $r$ th segment $P_{i}\left(u_{r-1}, u_{r}\right)$ of $P_{i}$ to $T P_{i-1}^{r-1}$ in order to form a new tree $T P_{i-1}^{r}$. Let $w=$ $L C A\left(u_{r-1}, u_{r}\right)$ be the lowest common ancestor of $u_{r-1}$ and $u_{r}$ in tree $T P_{i-1}^{r-1}$. Then, there are two node-disjoint paths in $T P_{i-1}^{r-1} \cup\left\{P_{i}\left(u_{r-1}, u_{r}\right)\right\}$ from $w$ to $u_{r}$, which can further be classified into three cases (see Fig 3 ).

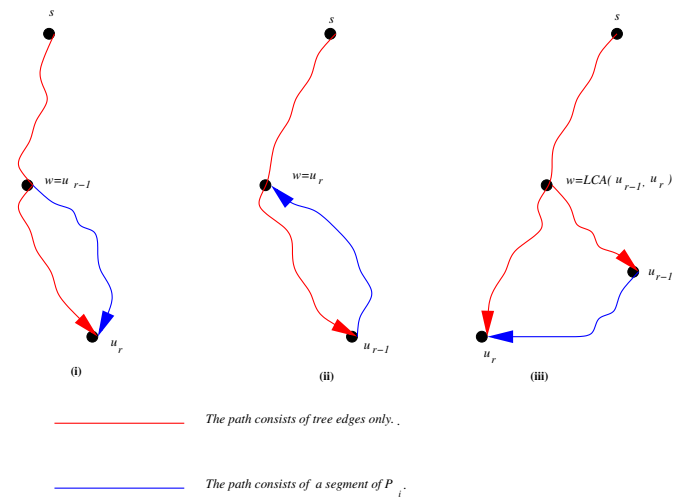

Figure 3. Add segment $P_{i}\left(u_{r-1}, u_{r}\right)$ to $T P_{i-1}^{r-1}$.

(i) $w=u_{r-1}$. In this case the two paths are $T P_{i-1}^{r-1}\left(u_{r-1}, u_{r}\right)$ consisting of the tree edges of $T P_{i-1}^{r-1}$, and $P_{i}\left(u_{r-1}, u_{r}\right)$. If $D\left(T P_{i-1}^{r-1}\left(u_{r-1}, u_{r}\right)\right) \leq$ $D\left(P_{i}\left(u_{r-1}, u_{r}\right)\right)$, we just ignore $P_{i}\left(u_{r-1}, u_{r}\right)$ for further consideration because the path delay in $T P_{i-1}^{r}$ from $s$ to $v_{i}$ is no more than $D\left(T P_{i-1}^{r-1}\left(s, u_{r-1}\right)\right)+D\left(T P_{i-1}^{r-1}\left(u_{r-1}, u_{r}\right)\right)+$ $D\left(P_{i}\left(u_{r}, v_{i}\right)\right) \leq D\left(T P_{i-1}^{r-1}\left(s, u_{r-1}\right)\right)+D\left(P_{i}\left(u_{r-1}, u_{r}\right)+\right.$ $P_{i}\left(u_{r}, v_{i}\right) \leq(1+\epsilon) \Delta$; otherwise, $T P_{i-1}^{r}$ is constructed by adding $P_{i}\left(u_{r-1}, u_{r}\right)$ to $T P_{i-1}^{r-1}$ and removing the tree edge $\left(u_{r}\right.$, parent $\left.\left(u_{r}\right)\right)$ from $T P_{i-1}^{r-1}$ at the same time. Now we see that the path delay in $T P_{i-1}^{r}$ from $s$ to each $v_{j}$ which is included in a subtree of $T P$ rooted at $u_{r}$ is no more than $(1+\epsilon) \Delta$, because $D\left(T P_{i-1}^{r-1}\left(s, u_{r-1}\right)\right)+D\left(P_{i}\left(u_{r-1}, u_{r}\right)\right)+$ $D\left(T P_{i-1}^{r-1}\left(u_{r}, v_{j}\right)\right) \leq D\left(T P_{i-1}^{r-1}\left(s, u_{r-1}\right)\right)+$ $D\left(T P_{i-1}\left(u_{r-1}, u_{r}\right)\right)+D\left(T P_{i-1}^{r-1}\left(u_{r}, v_{j}\right)\right)(1+\epsilon) \Delta$,
$1 \leq j \leq i-1$. (ii) $w=u_{r}$. In this case the path $P_{i}\left(u_{r-1}, u_{r}\right)$ will not be considered and $T P_{i-1}^{r}=T P_{i-1}^{r-1}$ because the path delay in $T P_{i-1}^{r}$ from $s$ to $v_{i}$ is $D\left(T P_{i-1}^{r-1}\left(s, u_{r}\right)\right)+D\left(P_{i}\left(u_{r}, v_{i}\right)\right) \leq$ $D\left(T P_{i-1}^{r-1}\left(s, u_{r-1}\right)\right)+D\left(P_{i}\left(u_{r-1}, u_{r}\right)\right)+D\left(P_{i}\left(u_{r}, v_{i}\right)\right) \leq$ $(1+\epsilon) \Delta$ due to the fact that $u_{r}$ is an ancestor of $u_{r-1}$ in $T P_{i-1}^{r-1}$. (iii) $w \neq u_{r-1}$ and $w \neq u_{r}$. There are two node-disjoint paths in $T_{i-1}^{r-1} \cup\left\{P_{i}\left(u_{r-1}, u_{r}\right\}\right.$ from $w$ to $u_{r}$. One is $T P_{i-1}^{r-1}\left(w, u_{r}\right)$ consisting of the tree edges only, and another is the joint of two paths $T P_{i-1}^{r-1}\left(w, u_{r-1}\right)$ consisting of tree edges only, and $P_{i}\left(u_{r-1}, u_{r}\right)$ at node $u_{r-1}$. If $D\left(T P_{i-1}^{r-1}\left(w, u_{r}\right)\right) \leq$ $D\left(T P_{i-1}^{r-1}\left(w, u_{r-1}\right)\right)+D\left(P_{i}\left(u_{r-1}, u_{r}\right)\right), \quad$ then $T P_{i-1}^{r}=T P_{i-1}^{r-1}$ and $P_{i}\left(u_{r-1}, u_{r}\right)$ will not be considered because the delay in $T_{i-1}^{r}$ from $s$ to $v_{i}$ is $D\left(T P_{i-1}^{r-1}(s, w)\right)+D\left(T P_{i-1}^{r-1}\left(w, u_{r}\right)\right)+D\left(P_{i}\left(u_{r}, v_{i}\right)\right) \leq$ $D\left(T P_{i-1}^{r-1}(s, w)\right)+D\left(T P_{i-1}^{r-1}\left(w, u_{r-1}\right)\right)+$ $D\left(P_{i}\left(u_{r-1}, u_{r}\right)\right)+D\left(P_{i}\left(u_{r}, v_{i}\right)\right) \leq(1+\epsilon) \Delta$. Otherwise, $T P_{i-1}^{r}$ is constructed by adding the path $P_{i}\left(u_{r-1}, u_{r}\right)$ to $T P_{i-1}^{r-1}$ and removing the edge $\left(u_{r}, \operatorname{parent}\left(u_{r}\right)\right)$ from it at the same time. It is not difficult to show that the path delay in $T P_{i-1}^{r}$ from $s$ to each node $v_{j}$ that is included in the subtree of $T P_{i-1}^{r-1}$ rooted at $u_{r}$, is no greater than $(1+\epsilon) \Delta$ due to the fact that $D\left(T P_{i-1}^{r-1}(s, w)\right)+$ $D\left(T P_{i-1}^{r-1}\left(w, u_{r-1}\right)\right)+D\left(P_{i}\left(u_{r-1}, u_{r}\right)\right)+$ $D\left(T P_{i-1}^{r-1}\left(u_{r}, v_{j}\right)\right) \leq D\left(T P_{i-1}^{r-1}(s, w)\right)+$ $D\left(T P_{i-1}^{r-1}\left(w, u_{r}\right)\right)+D\left(T P_{i-1}^{r-1}\left(u_{r}, v_{j}\right)\right) \leq(1+\epsilon) \Delta$, $1 \leq j \leq i-1$ and $1 \leq i \leq\left|D_{i_{j}}\right|$.

When the last segment (assuming the $r$ th segment) of $P_{i}$ has been considered, the resulting tree is a directed tree $T P_{i}=T P_{i-1}^{r}$ rooted at $s$ including all nodes in $\left\{v_{1}, v_{2}, \ldots, v_{r}\right\}\left(\subseteq D_{i_{j}}\right)$, and the path delay in $T P_{i}$ from $s$ to $v_{j}$ is no more than $(1+\epsilon) \Delta$ for all $j, 1 \leq j \leq$ $r$, and $C\left(T P_{i}\right) \leq C\left(T P_{i-1}\right)+C\left(P_{a p p}\left(s, v_{i}, \lambda_{i_{j}}\right)\right) \leq$ $\sum_{x=1}^{i} C\left(P_{a p p}\left(s, v_{x}, \lambda_{i_{j}}\right)\right), 1 \leq x \leq\left|D_{i_{j}}\right|$. We therefore have the following lemma.

Lemma 3 Let $T_{j}$ be the light multicast tree in $G_{\lambda_{i_{j}}}$ rooted at $s$ including the nodes in $D_{i_{j}}$, constructed by the sticking approach, and $C\left(T_{j}\right)$ be the cost of $T_{j}$. Let $C^{*}$ be the optimal cost of the MDCLMT, Then, $C\left(T_{j}\right) \leq\left|D_{i_{j}}\right| C^{*}$. It takes $O\left(\left|D_{i_{j}}\right| n^{2}\right)$ time to construct $T_{j}$.

Proof Following the similar argument as Lemma 2, it is easy to see that $C\left(P_{a p p}\left(s, v_{x}, \lambda_{i_{j}}\right)\right) \leq C^{*}$ with the path delay $(1+\epsilon) \Delta$ for all $v_{x} \in D_{i_{j}}$. Let $T P_{i}$ be the tree after adding the path $P_{a p p}\left(s, v_{i}, \lambda_{\lambda_{i_{j}}}\right)$ to tree $T P_{i-1}$. Following the construction of $T P_{i}$ from $T P_{i-1}$, it is obvious that $C\left(T P_{i}\right) \leq C\left(T P_{i-1}\right)+C\left(P_{a p p}\left(s, v_{i}, \lambda_{i_{j}}\right)\right) \leq i C^{*}$, $1 \leq i \leq l$. Thus, $C\left(T_{j}\right)=C\left(T P_{l}\right) \leq l C^{*}=\left|D_{i_{j}}\right| C^{*}$.

We now analyze the time complexity of the sticking approach. Assume that the path $P_{i}$ consists of $r$ segments. To obtain the tree $T P_{i}$, there are $r$ stages. At stage $r^{\prime}$, the $r^{\prime}$ th 
segment of $P_{i}$ is considered, and processing this segment involves finding a lowest common ancestor in tree $T_{i-1}^{r^{\prime}-1}$ for a pair of nodes and determining whether or not to add this segment to $T_{i-1}^{r^{\prime}-1}$, which takes $O(n)$ time. Thus, it takes $O\left(r^{\prime} n\right)$ time to construct $T P_{i}$. When $r^{\prime}=O(n)$ in the worst case, it takes $O\left(n^{2}\right)$ time to construct $T P_{i}$ from $T P_{i-1}$. There are $\left|D_{i_{j}}\right|$ paths in $G_{\lambda_{i_{j}}}$ are to be considered. As a result, the construction of a light multicast tree $T_{j}$ with wavelength $\lambda_{i_{j}}$ takes $O\left(\left|D_{i_{j}}\right| n^{2}\right)$ time.

We thus have the following theorem.

Theorem 5 Given a directed network $G=(V, E, \Lambda)$ with a given source $s$ and $a$ set $D$ of destination nodes, and an integral delay $\Delta$, assume that each link $e \in E$ of $G$ has been assigned a set $\Lambda(e)(\subseteq \Lambda)$ of wavelengths, each wavelength $\lambda \in \Lambda(e)$ traversing e incurs a cost $w(e, \lambda)$, and the delay of traversing $e$ is an integer $\delta(e)(\leq \Delta)$. There is an approximation algorithm for the MDCLMT with a performance ratio of $|D|$ and the path delay of $(1+\epsilon) \Delta$. The time complexity of the proposed algorithm is $O\left(k m n / \epsilon+|D| n^{2}\right)$, where $\epsilon$ is constant, $0<\epsilon \leq 1$.

Proof Following Lemma 3, the solution delivered by the proposed algorithm is $\sum_{j=1}^{k^{\prime}} C\left(T_{j}\right) \leq \sum_{j=1}^{k^{\prime}}\left|D_{i_{j}}\right| C^{*}=$ $|D| C^{*}$.

The running time of the proposed algorithm is analyzed as follows. To partition the nodes in $D$ into $k^{\prime}$ disjoint subsets $D_{i_{j}}$, apply the Goel et al [4] algorithm for each induced graph $G_{\lambda}$ for any $\lambda \in \Lambda, 1 \leq i_{j} \leq k, 1 \leq j \leq k^{\prime} \leq k$. Note that the Goel et al algorithm [4] takes $O(m n / \epsilon)$ time on each graph $G_{\lambda}$ for each $\lambda \in \Lambda$. Thus, the time used for the partition is $O(k m n / \epsilon)$. Then, apply the sticking approach to each subset to construct a light multicast tree. As results, the $k^{\prime}$ light multicast trees are constructed, which form a solution for the MDCLMT. The time used for this step is $O\left(\sum_{j=1}^{k^{\prime}}\left|D_{i_{j}}\right| n^{2}\right)=O\left(|D| n^{2}\right)$, following Lemma 3. The theorem then follows.

\section{Acknowledgment}

It is acknowledged that the work by Weifa Liang was supported by a research grant \#DP0449431, funded by Australian Research Council under its Discovery Schemes.

\section{References}

[1] M. Charikar, C. Chekuri, T-Y Cheung, Z. Dai, A. Goel, S. Guha and M. Li. Approximation algorithms for directed Steiner problems. J. Algorithms, Vol. 33, 73-91, 1999.

[2] I. Chlamtac, A. Faragó, and T. Zhang. Lightpath (wavelength) routing in large WDM networks. IEEE Journal on Selected Areas in Communications, Vol.14, 909-913, 1996.
[3] M. R. Garey and D. S. Johnson. Computers and Intractability: A guide to the Theory of NPCompleteness. W.H. Freeman and Company, NY, 1979.

[4] A. Goel, K.G. Ramakrishnan, D. Kataria, and D. Logothetis. Efficient computation of delay-sensitive routes from one source to all destinations. Proc. of INFOCOM'01, 2001.

[5] V. P. Kompella, J. C. Pasquale, and G. C. Polyzos. Multicast routing for multimedia communication. IEEE/ACM Trans. Networking, Vol. 1, pp. 286-292, 1993.

[6] W. Liang and H. Shen. Multicasting and broadcasting in large WDM networks. Proc. of 12th Int'l Conf. on Parallel Processing Symp., IEEE Computer Society Press, 1998, 365-369.

[7] W. Liang and $X$. Shen. Improved lightpath (wavelength) routing in large WDM networks. IEEE Trans. Commun., Vol.48, 1571-1579, 2000.

[8] R. Libeskind-Hadas. Efficient collective communication in WDM networks. Proc. of $I C^{3} N, 2000,612-$ 616.

[9] R. Malli, X. Zhang and C. Qiao. Benefit of multicasting in large WDM networks. Proc. of SPIE Conf. on All-Optical Networks, Vol 3531, 1998, 209-220.

[10] B. Mukherjee. WDM-based local lightwave networks, Part I: single-hop systems. IEEE Network, Vol.6 (3), 12-27, 1992.

[11] B. Mukherjee. WDM-based local lightwave networks, Part I: multi-hop systems. IEEE Network, Vol.6 (4), 20-32, 1992.

[12] R. K. Pankaj. Wavelength requirements for multicasting in all-optical networks. IEEE/ACM Trans. Networking, Vol.7, 414-424, 1999.

[13] L. H. Sahasrabudde and B. Mukherjee. Light-trees: optical multicasting for improved performance in wavelength-routed networks. IEEE Communication Magazine, Vol.32, 67-73, 1999.

[14] G. Sahin and M. Azizoglu. Multicast routing and wavelength assignment in wide-area networks Proc. of SPIE Conf. on All-Optical Networks, Vol 3531, 1998, 196-208.

[15] J. Yates, M. Rumsewicz and J. Lacey. Wavelength converters in dynamically reconfigurable WDM networks. IEEE Communication Surveys, 1999.

[16] X. Zhang, J. Wei and C. Qiao. Constrained multicast routing in WDM networks with sparse light splitting. Proc. of IEEE INFOCOM'00, 2000, 1781-1790.

[17] T. F. Znati, T. Alrabiah and R. Melhem. Low-cost delay-bounded point-to-point multipoint communication to support multicasting over WDM networks. Computer Networks, Vol.38, 423-445, 2002. 